C2008 IEEE. Personal use of this material is permitted. However, permission to reprint/republish this material for advertising or promotional purposes or for creating new collective works for resale or redistribution to servers or lists, or to reuse any copyrighted component of this work in other works must be obtained from the IEEE. 


\title{
Developing a Semantic Service Transaction System in the DE Environment
}

\author{
Hai Dong, Farookh Khadeer Hussain, Elizabeth Chang \\ Digital Ecosystem and Business Intelligence Institute \\ Curtin University of Technology \\ E-mail: \{hai.dong, farookh.hussain, elizabeth.chang\}@cbs.curtin.edu.au
}

\begin{abstract}
Digital Ecosystem (DE), which is an emerging service environment beyond the service-oriented architecture (SOA), is increasingly attracting researchers' interest. However, current literature reveals that there is not reliable and trustworthy technical support for service transaction activities in the DE environment. In this paper, we propose a semantic service transaction system - SST, in order to provide the reliable and trustworthy services in the aspects of service search, negotiation, contract and feedback.
\end{abstract}

\section{INTRODUCTION}

Digital Ecosystem (DE) is increasingly attracting the interest of researchers [9]. However the current literature reveals that there is no reliable and trustworthy technical support in order to carry out service transactions and associated activities in the DE environment. In this paper, we propose a semantic service transaction system - SST. SST would provide a platform to carry out reliable and trustworthy services in the aspects of service search, negotiation, contract and feedback.

In the latter part of this paper, we will introduce the architecture of system. However, before that, we intend to carry out a rough review on the literal researches with respect to the field of DE, semantic search and negotiation ontologies, followed by the analysis of issues in these fields. In the fourth section of this paper, we will draw the overall framework of SST system. The further detailed working of the SST system will be described in the fifth section. Then the present status of prototype implementation will then be summarized. We will draw a conclusion to the current work and plan future works in the final section.

\section{LITERATURE REVIEW}

In this section, we will briefly review the literature regarding digital ecosystems, semantic search engines and negotiation ontologies.

\section{A. Digital Ecosystems}

DE is defined as "an open, loosely coupled, domain clustered, demand-driven, self-organizing and agent-based environment, which each species is proactive and responsive for its own benefit and profit" [5] [6], which is a neoteric terminology and its appearance is as a result of the natural existence of business ecosystem, along with the evolution of business, e-business, business networks and information technology. The goal of DE is to improve the efficiency of the communication between internal agents and to structuralize the existing business ecosystem [14]. The contemporary DE researches focus on theoretical study and application development [8]. DE is composed of two basic elements: species and environments [6]. Species are mainly categorized into three types: biological species, economic species and digital species. Species can play dual roles in the digital ecosystem, namely service requester (client) - that needs services, and service provider (host) - that provides services.

Small and medium enterprises (SMEs) are one of the major targets which DE serves [5]. SMEs are regarded as one of the major power promoting the economic growth of the world [1]. SMEs urgently need collaboration rather than competition with the assistance of ICT. This is due to their features of fragility, small size and the lack of capability, power and speed [4].

\section{B. Semantic Search Engines}

Guha et al. categorize the existing search engines into two categories - navigational search and research search. In the first category, users enter keywords, which cannot be used to denote concepts, to query documents where the keywords coexist. In the second category, users use words to denote certain concepts, which could help search engines to understand users' query intentions. Semantic search concentrates on the second form of search [11].

Currently, many semantic search engines are designed and implemented in order to adapt to different working environments, and the mechanisms that realize these search engines are thus distinct. Guha and McCool deliver a semantic search engine in TAP system - a comprehensive semantic web system [10]. The query language for semantic search in TAP is called GetData interface, which allows programs to visit properties of a resource in a semantic graph. Liu, Shen and Liao invent an e-service platform integrated with semantic search for e-service metadata [12]. E-service metadata refers to descriptions about e-services and providers, which is to publish and to discover e-services. There are two types of metadata in the system: business level metadata - the description of e-service providers; service level metadata - the description of basic information about eservice. The authors adopt Universal Description, Discovery 
and Integration which is a web service standard to register and search e-services. Dichev and Dicheva propose a viewbased semantic search engine in the context of topic-centered learning repository, by means of the extension of the Topic Maps (TM) model - which is a lightweight ontology model constructed by topics and relationships between topics [7]. Wang et al. project a semantic search methodology to retrieve information from normal tables, which has three main steps: identifying semantic relationships between table cells; converting tables into data in the form of database; retrieving objective data by query languages [17].

\section{Negotiation Ontology}

Negotiation is defined as two or more agents making agreement on the common interests [2] [16]. In the last few years, negotiation research has gained increasing attentions from researchers in the field of business and computer science. The current negotiation research focuses on negotiation protocols and negotiation strategies. The former mainly refers to generating rules and regulations for new participants to adapt to negotiation environments; the latter refers to the principles about selecting different actions to respond in different negotiation scenarios [16]. Negotiation ontology is not a new topic and most negotiation ontologies are designed for automatically generating protocols for the negotiation behavior in e-business [2] [13] [15] [16].

Tamma proposes an ontology for automated negotiation process in context of e-commerce. By London classifications, Tamma identifies the common characteristics of most negotiation protocols. Based on the characteristics, Tamma creates a negotiation ontology which shares a common vocabulary to support the automated negotiation process regardless of any negotiation mechanism. By means of this ontology, the agent will choose the most suitable protocols for the given negotiation contents [16].

In addition, some researchers attempt to solve the issues existing in the negotiation process, in terms of ontology engineering. Bravo focuses on the incompatibility of protocols between different negotiating ontologies, and designs a new ontology involving a shared vocabulary of negotiation terms and messages as well as a semantic disambiguation module, to release the misunderstandings between agents with different protocol ontologies [2]. Similarly, Malucelli also recognizes the semantic heterogeneity issues in negotiation process. In the traditional negotiation ontologies, when a new agent wants to participate in a negotiation process, it has to be reprogrammed to suit to the protocol existing in the process. In order to address the issue, Malucelli utilizes the JADE (Java Agent Development Framework) platform and OWL (Web Ontology Language) to construct a two-double ontological architecture. The toplevel ontology contains shared negotiation terms and messages for all agents. For the specific negotiation, the agent is coded with the domain-specific ontology. Thus, this design can be seen as a solution for the semantic heterogeneity problem in negotiation process [13].

\section{RESEARCH ISSUES}

In DE, as the main customer group, SMEs do not have the ability to build network infrastructure and relevant platform softwares by themselves. However, they indeed have the urgent requirement to gain competitiveness in their industries [1]. Thus, on one hand ICT (Information and Communication Technology) providers needs to support the DE services to SMEs; on the other hand, by means of the DE services, SMEs are able to search interested services to improve their competitiveness. However, there is not such a service search engine available in the DE environment.

Meanwhile, SMEs are regarded as the service requestors, ICT providers and other services providers are regarded as the service providers, negotiation activities are occurring in the process of mutual contact and subsequent interactions. Owing to the reason that service provider and service requestor may possibly not be in the same industry, some issues may appear that misunderstandings on terms and other messages exist in the process of information exchange, which can slower the speed of service providing and thereby reducing the competitiveness of SMEs. Ontology, as the primary means of interdisciplinary knowledge sharing, can be utilized to solve the issue. Hence, a negotiation ontology for service in DE domain is urgently needed.

In addition, the foundation of a transaction is built upon the mutual trust of two entities in the transaction. This sort of trust heavily relies on the reputation of service providers. To quantify and subsequently use the reputation would be an objective approach. Therefore, we need a feedback system which allows service requesters to evaluate the reputation of service providers once the transaction is completed.

\section{SST CONCEPTUAL MOdEL}

The conceptual model of the proposed SST system consists of four phases as shown in Fig. 1, which are service search phase, negotiation phase, contract phase and feedback phase.

In the service search phase, a customized semantic service search engine is designed, in order to assist service requesters to query proper services from a service knowledge base. In the negotiation phase, according to the retrieved services, a multi-domain service negotiation ontology is constructed, with the purpose of providing protocols for negotiations between service requester agents and service provider agents. Once both the entities involved in an interaction come to an agreement, all the negotiation details which are stored in the form of negotiation metadata are then transmitted to the contract phase, which conducts a formal contract based on a contract ontology. To measure the reputation of service

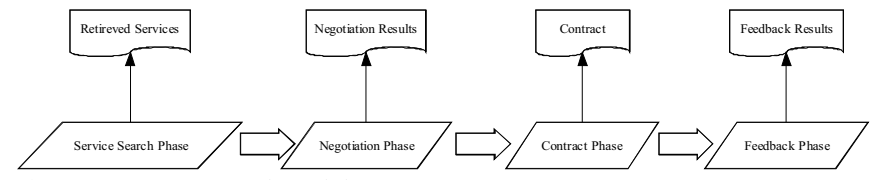

Fig. 1. SST conceptual model 
providers, after a service is delivered, service requester is required to provide comments to the feedback system that bases on a feedback ontology.

\section{Detailed Process of SST System}

In this section, we will briefly describe the further processes of the proposed SST system.

\section{A. Service Search Phase}

Service search phase is the key element of the SST system. Two main components are involved in this phase, which are a service knowledge base and a semantic search engine (Fig. 2).

First of all, in order to abstract the service entities in the DE environment, a standard metadata format - Service Description Entity (SDE) format is designed, in Web Ontology Language (OWL). By means of the SDE format, SDE metadata are abstracted from online service descriptions, and are then stored in a service knowledge base. Meanwhile, these SDE metadata are identified by their unique Uniform Resource Identifiers (URIs). To cluster the SDE metadata, we build a service ontology working in multiple domains. Based on the semantic matching between service descriptions of ontological concepts and the descriptions regarding SDE metadata, the two parts are semantically related.

In the search engine, a semantic search algorithm is utilized for users to query relevant services based on keywords. The search engine will adopt an interactive Graphic User Interface (GUI) which benefits users to precisely denote the ontological concepts which are close to the query. Along with the denotation of ontological concepts, the SDE metadata which are semantically relevant to the concepts are displayed to users.

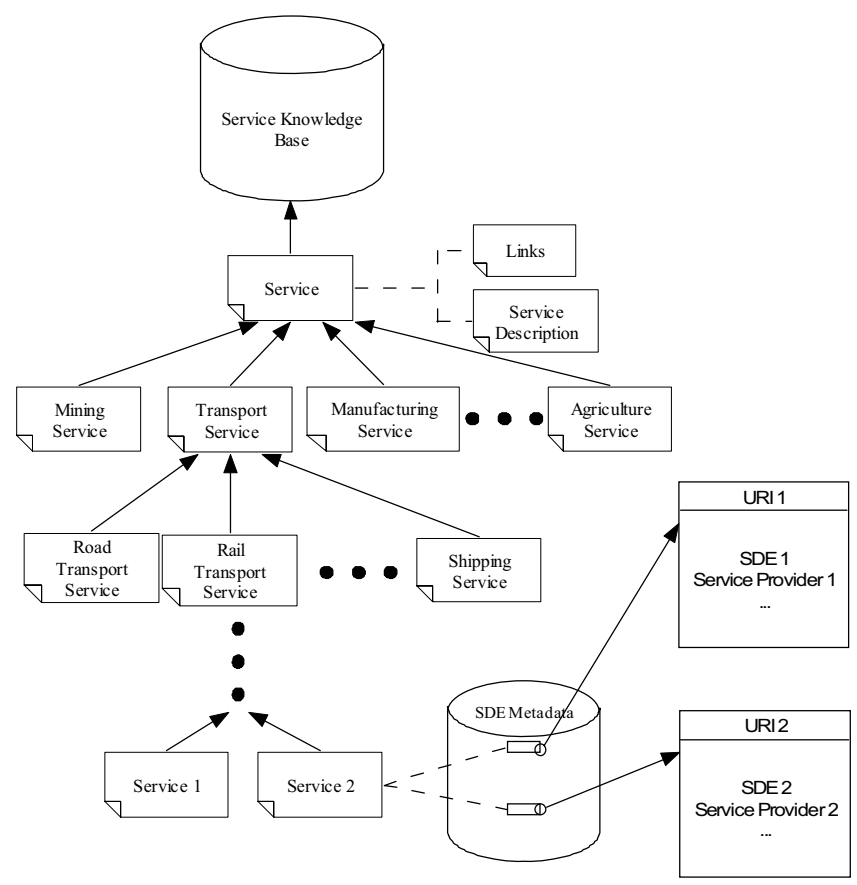

Fig. 2. Service ontology and SDE metadata

\section{B. Negotiation Phase}

After a group of SDE metadata is retrieved in the service search phase, according to the metadata content, the service providers who are relevant to the metadata are located. At this stage the user can select the service providers with the highest reputation values, which can be measured in the feedback phase. Once the user determines a service provider, s/he then can enter into the negotiation phase with the service provider. The essence of negotiation system is built upon a multidomain service negotiation ontology (Fig. 3). The service negotiation ontology is able to generate domain-specific negotiation rules and regulations for user according to the actual service domain. Here the service domain is decided by the concept selected by user, which is relevant to the SDE metadata. The rules and regulations are able to form a series of standard negotiation steps, which can be followed by service requester (users) and service providers. In these steps, all negotiation details between two parts are stored in the form of negotiation metadata.

\section{Contract Phase}

Once an agreement is conducted between two parts in the negotiation process, the details of agreement namely the negotiation metadata is then transmitted into the contract phase. In this phase, a service contract ontology is designed, which is based on the multi-domain service negotiation ontology. The result of this phase is to phrase and form a formal contract document between service providers and service requesters.

\section{Feedback Phase}

As mentioned in the service search phase, when user denotes a concept in the service ontology, there are usually numerous SDE metadata linked to the concept. To choose the proper SDE metadata from them, service providers' reputation values can be utilized as a primary criterion. The reputation values are evaluated in this phase. The primary component of feedback phase is a feedback ontology (Fig. 4). In the feedback ontology, after each service transaction is completed, service requester is required to give feedbacks to the quality of provided services. We adopt the CCCI Metrics to allow service requesters to evaluate services from quantitive perspective [4]. By means of the CCCI Metrics, the trustworthiness of a service can be measured. By normalizing the trustworthiness values of all services provided by a

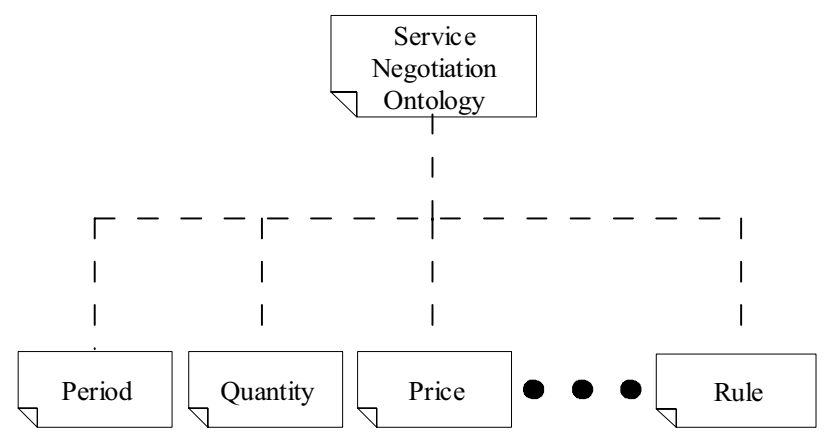

Fig. 3. Service negotiation ontology 


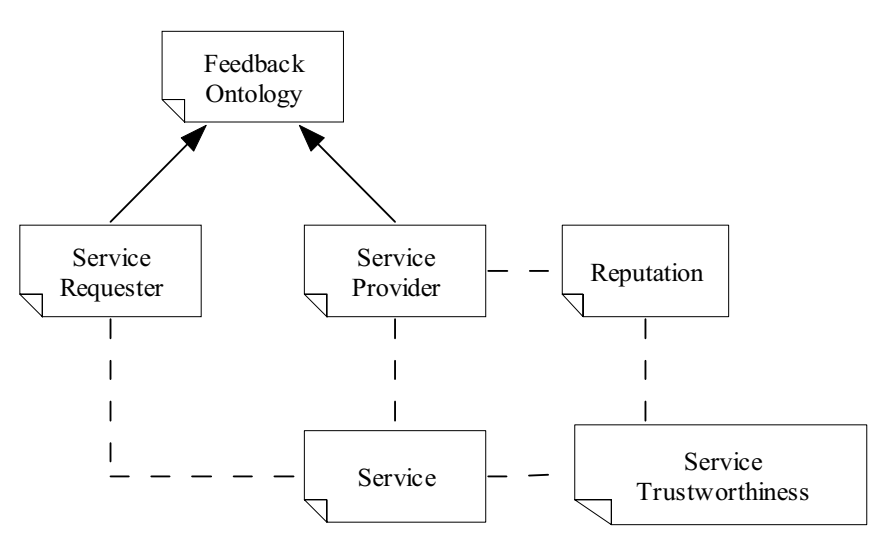

Fig. 4. Feedback ontology

service provider, the service provider's reputation value can be measured.

\section{PROTOTYPE IMPLEMENTATION}

Currently we are implementing the semantic search engine in the first phase. OWL and JAVA are the primary programming languages adopted in this project. Transport domain is chosen as where the domain-specific ontology is mapped. We design a semantic crawler to extract SDE metadata from the Australian Yellow Pages $(\mathcal{C}$ website. A semantic search algorithm evolved from Case-based Reasoning (CBR) algorithm is adopted in the search engine [3]. In addition, we make use of Wordnet as the query filtering and expansion approach.

We design an interactive search GUI (Fig. 5). The GUI can display the hierarchical structure of ontological concepts. Once user enters keywords, the semantic similarity value of each concept associated with the keywords is measured, and the matched concepts are ranked based on the values. If there are subconcepts under a matched concept, these subconcepts can be unfolded as well. This design can benefit users to precisely denote the ontological concepts that are semantically relevant to their query words. Users then can denote the concepts they prefer. For each denoted concept, all its semantically relevant SDE metadata are displayed to users.

\section{CONCLUSION AND FUTURE WORKS}

In this paper we present the framework of a semantic service transaction system - SST, in order to provide a reliable and trustworthy approach for the service transaction activities in the DE environment. From the literature it can be

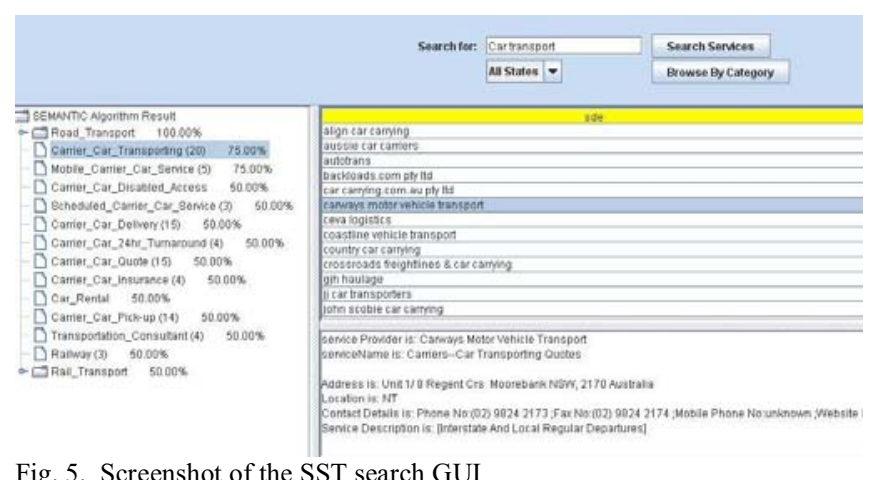

Fig. 5. Screenshot of the SST search GUI observed that there is no existing methodology available for the transaction activities in the emerging DE environment. Therefore a service transaction system is urgently required in this field.

The system is composed of four major design phases. The first is search phase which contains a service knowledge base and a semantic search engine. The service knowledge base stores the SDE metadata which are abstracted from the services descriptions on the internet. Here a domain-specific service ontology is designed, in order to cluster the SDE metadata. Additionally a semantic search algorithm is adopted to realize the semantic queries for SDE metadata. The second is negotiation phase. For a retrieved SDE metadata, the service provider who refers to the metadata can be observed. An ontology-based negotiation system can be used as the negotiation tool between two parts in the transaction. When both of the negotiation parts come to an agreement, the negotiation details namely negotiation metadata are then transferred into the third phase - contract, which can form a formal contract for the service transaction. After a service is delivered, service requesters can measure the trustworthiness of services by the fourth phase feedback. Moreover, the trustworthiness value of a service can influence the reputation value of its provider. The service providers' reputation values can be used as an efficient measure which allows service requesters to choose the most proper service providers in the negotiation phase.

The implementation of SST system is an ongoing project in which the negotiation, contract and feedback phase will be finished in the future. For the realization of negotiation system, we compare and contrast the available negotiation ontologies, and combine them to satisfy the featured requirement of DE environment. For the feedback phase, a feedback ontology for universal domains is being designed.

\section{ACKNOWLEDGMENT}

We would like to express our gratitude to the assistance of DEBII.

\section{REFERENCES}

[1] "The Digital Ecosystems Research Vision: 2010 and Beyond," Digital Business Ecosystem, 2005.

[2] M. C. Bravo, J. Pérez, V. J. Sosa, A. Montes, and G. Reyes, "Ontology Support for Communicating Agents in Negotiation Processes," in Proceedings of the Fifth International Conference on Hybrid Intelligent Systems, Rio de Janeiro, 2005, pp. 482 - 487.

[3] D. C. J. Carthy, A. Drummond, J. Dunnion, and J. Sheppard, "The use of data mining in the design and implementation of an incident report retrieval system," in Systems and Information Engineering Design Symposium, Charlottesville, 2003, pp. 13-18.

[4] E. Chang, T. Dillon, and F. Hussain, Trust and Reputation for Service Oriented Environments-Technologies for Building Business Intelligence and Consumer Confidence: John Wiley \& Sons, 2005.

[5] E. Chang, M. Quaddus, and R. Ramaseshan, "The vision of DEBI Institute: digital ecosystems and business intelligence: Digital Ecosystem and Business Intelligence Institute," DEBII, Perth 2006.

[6] E. Chang and M. West, "Digital Ecosystem - A next generation of the collaborative environment," in iiWAS2006, Yogyakarta, 2006.

[7] C. Dichev and D. Dicheva, "View-Based Semantic Search and Browsing " in $W I$ '06 2006. 
[8] P. Dini, "Structure and outlook of digital ecosystem research," in IEEE DEST 2007, Cairns, 2007.

[9] P. Ferronatoi, "Architecture for Digital Ecosystems, beyond Service Oriented Architecture," in IEEE DEST 2007, Cairns, 2007, pp. 660 665.

[10] R. Guha and R. McCool, "TAP: a Semantic Web platform," Computer Networks, vol. 42, pp. 557-577, 2003.

[11] R. Guha, R. McCool, and E. Miller, "Semantic search." vol. 2008: W3C, 2003.

[12] D.-R. Liu, M. Shen, and C.-T. Liao, "Designing a composite e-service platform with recommendation function," Computer Standards \& Interfaces, vol. 25, pp. 103-117, 2003

[13] A. Malucelli, D. Palzer, and E. ä. Oliveira, "Ontology-based services to help solving the heterogeneity problem in e-commerce negotiations,"
Electronic Commerce Research and Applications, vol. 5, pp. 29-43, 4 October 20062006.

[14] F. Nachira, "Innovation Ecosystem: una strategia europea l'innovazione e lo sviluppo economico per," Creative Commons 2006.

[15] M. Schoop, M. U. Rehman, and A. Jertila, "Specification of agent technology for negotiation support," 2004.

[16] V. Tamma, S. Phelps, I. Dickinson, and M. Wooldridge, "Ontologies for supporting negotiation in e-commerce," Engineering Applications of Artificial Intelligence, vol. 18, pp. 223-236, 5 October 20062005.

[17] H. L. Wang, S. H. Wu, I. C. Wang, C. L. Sung, W. L. Hsu, and W. K. Shih, "Semantic search on Internet tabular information extraction for answering queries " in CIKM' 00 McLean, 2000, pp. 243-249. 